\title{
BUILDINGS STRUCTURE RESPONSE DUE TO RAILWAY TRAFFIC
}

The free-field dynamic response at the distance and dynamic response of building structures due to railway traffic calculation procedures is described in this paper. The viscoelastic halfspace model is used both for evaluation of the track-soil interaction forces as well as for prediction of the ground-borne vibrations spectral functions at the distance. In the next step these functions are applied for building structure dynamic response calculation via relevant computational building structure model.

Keywords: Microtremor, railway traffic effects on structures, prediction dynamic half space and structures response models, in situ experimental tests, spectral analysis.

\section{Introduction}

The growing traffic volume, the higher population density and the diminishing distance between the track and the structure can be considered to be responsible for increasing vibration nuisance due to railway traffic. Therefore, the development and validation of a numerical prediction model for traffic induced vibrations in buildings is treated in many works. Empirical models show a close relationship to a set of experimental data but the application of the model is limited to similar conditions. Also these models do not always provide insight in the influence of specific parameters. Numerical models allow the influence of various parameters to be investigated but a validation of the model with experimental data is required to verify the underlying theoretical assumptions. Even though the validation focuses on traffic induced vibrations, the numerical prediction model can be generally applicable to other types of vibration sources [1]. The dynamic train-track interaction is a coupled problem, contrary to vehicle-road interaction problems, that requires the simultaneous solution of the equations of motion of the train and the track. The train-track interaction forces due to the track unevenness are computed using a flexibility formulation. A two-dimensional linear vehicle model with a limited number of DOF is coupled to a linear elastic longitudinal invariant track model, which allows a solution of the equations of motion in the frequency-wavenumber domain [2]. The transfer functions between the track and the soil and the computed interaction forces are used to compute the response at any arbitrary point in the free field [3-5]. Finally, the building structure dynamic response at the distance calculation is performed using half - space output data (PSD, time history) as the input data into the building structure foundations [6].

\section{Track model description - numerical approach}

A numerical prediction model for ground-borne vibrations due to railway traffic on ballasted track requires the modelling of several components, as indicated in Fig. 1. This paper presents a numerical prediction model which calculates the ground-borne vibration level due to railway traffic in two steps. The first step determines the dynamic track-soil interaction forces using a detailed train model and the dynamic behaviour of the layered spring-damper system and the through-soil coupling of the sleepers are accounted for the soil model [7-9].

The prediction of the ground-borne vibration level at the distance in the second step is based on the viscous-elastics soil model $[1,2,10,11,12]$.

The vehicle car-body, the bogie and the wheelset are modelled as rigid bodies connected by springs and dampers, Fig. 2e. The wheelset is connected to the rail with a linearized Hertzian spring. The rail is modelled as a hinged Rayleigh beam with rotational inertia. In the track model the rail is supported discretely by sleepers modelled as rigid bodies with spring-damper systems representing the railpads. The sleeper is modelled as a short Rayleigh beam resting on flexural mass layer supported by discretely Pasternak spring-damper systems representing the elastic and attenuation characteristics of the railway ballast and substrate soils. As a result, the model evaluates the track-soil interaction forces in terms of the spectral density function which is often calculated as the statistical description of the rail roughness function [13]. This calculations followed by a second step in which the spectral density of the level of ground-borne vibrations is determined by FRF

\footnotetext{
* Jan Bencat ${ }^{1}$, Krzysztof Stypula ${ }^{2}$

${ }^{1}$ Civil Engineering Faculty, University of Zilina, Slovakia, E-mail: jan.bencat@gmail.com

${ }^{2}$ Civil Engineering Faculty, Cracow University of Technology, Cracow, Poland
} 


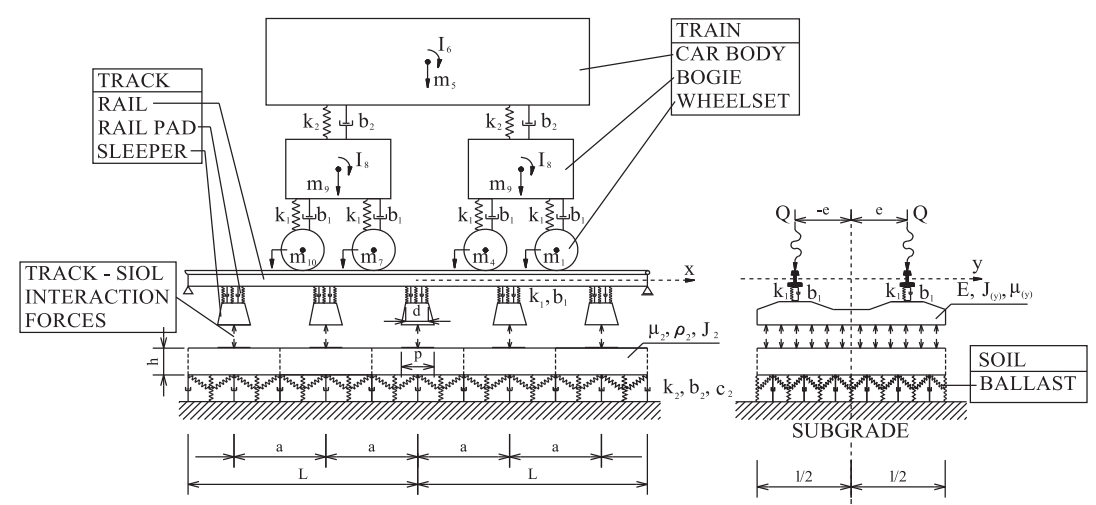

Fig. 1 Components of the train-track-soil system

between track and unbounded soil. Proposed prediction theoretical model for vertical track vibration numerical program consists of three parts:

- model of vehicle

- model of train-track interaction

- model of track (sleepers/ballast and subsoil).

The frequency characteristics method (input-output) was used for calculation modelled feedback linear dynamic train-track-soil system parameters, Fig. 2g (program Interaction). Final products of the numerical calculations are: vehicle, rail, sleeper, railpads and ballast frequency response functions (also sleepers deflection and bending moment in time domain) using spectral density functions (SDF) of the rail roughness used by railway operators or experimentally measured in situ for case study. An important example of non-linear behaviour is the wheel-rail contact but also the railpads and the suspension of the train can deflect in a nonlinear manner. Nevertheless the results presented in this study are limited to linear analyses. Also in this model is accepted symmetrical dynamic response of the sleepers to longitudinal axis of the track (rail roughness coherence function for left and right rail is equal to $\approx 1)$.

\subsection{Track model}

The track model commonly found in the literature represents the rail as infinite Timoshenko, Euler or Rayleigh beam [7-9] on a continuous uniform support, Fig. 2a. The beam is taken as uniform flexural rigidity $E I$, rail mass per unit $m_{r}$ and distributed sleeper mass $m_{s}$. The railpad stiffness and viscous damping constant per unit length are taken to be $k_{1}$ and $b_{1}$, respectively; the corresponding parameters for ballast are $k_{2}$ and $b_{2}$. An harmonic point force $p(t)=P \cos \omega t$ is assumed to run at constant velocity $v$ along the rail. The FRF of the track excited to a harmonic force for proposed prediction model are discussed in this section. The track model consists of two parts: (i) model of sleeper with ballast and subballast, Fig. $2 \mathrm{~b}$ and (ii) track model (rail supported discretely by sleepers modelled as rigid bodies with spring-damper systems representing the railpads), Fig. 2c, d.
Finally, the solution for FRF of the linear dynamic system model, e.g. in which on input are rail roughness $\xi$ and on output are wheel forces $Q$, enable calculations of the interaction matrix FRF according to scheme as shown in Fig. 2g. The dynamic displacement of the wheel $z$ is defined by $z=\xi+v+\eta$, where $\xi$ represents rail roughness, $v$ - rail vertical deflection and $\eta$ - wheel and rail contact deformation in contact location.

The track irregularities are great source of the track and vehicles dynamic excitations. Such excitation arises from discrete irregularities such as wheel flats and rail joints as well as periodic irregularities such as corrugation of the railhead. It is assumed that excitation of the track arises from a wheel passing over a sinusoidal irregularity on the rail head, (Fig. 2f). The stochastic theory analysis [3] enables to define irregularities by PSD function by $S_{\xi}(\Omega)=$ $=A \Omega^{-a}$ where $A$ and $a$ are empirical (experimental) constants. The distance $\mathrm{x}$ is used as the independent variable to define $\xi(x)$.

\subsection{Calculation of track component arbitrary FRF}

The frequency response function of arbitrary dynamic system part is calculated by rule of FRF summing as follows $W_{O}^{I}=W_{O 1}^{I}$. . $W_{O 2}^{O 1} . W_{O 3}^{O 2} \ldots \ldots \ldots . . W_{O i+1}^{O i} \ldots \ldots . W_{O}^{O n}$. The same way is used for summing of the arbitrary FRF matrix. E.g. consider a dynamic system with a defined input rail roughness $\xi$ producing a defined output sleeper deflection $v_{2}$, then the FRF is given by [13]:

$$
W_{v_{2}}^{\xi}=W_{Q}^{\xi} \cdot W_{Q_{1}}^{Q} \cdot W_{v_{2}}^{Q_{1}}
$$

\subsection{The response of track resting on a continuous rail supports}

An advantage of the continuous track model (Fig. 2c,d) is that enables for arbitrary track variables to calculate [13]: FRF, input spectra and standard deviation of the track dynamic response parameters - dynamic forces $Q, Q_{1}, Q_{2}$, dynamic deflections $v_{1}, v_{2}$, wheel centre dynamic displacement $\mathrm{z}$ and dynamic bending moment in rail and sleeper. As an example in Fig. 3 is plot of numerical 
a)

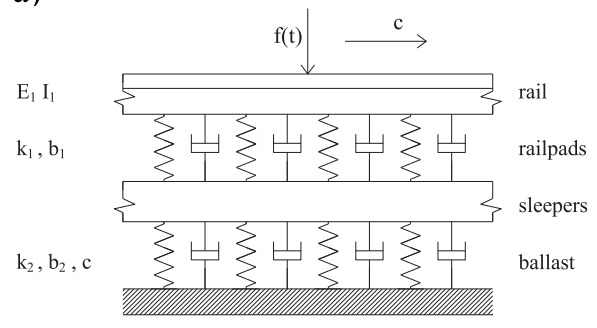

c)

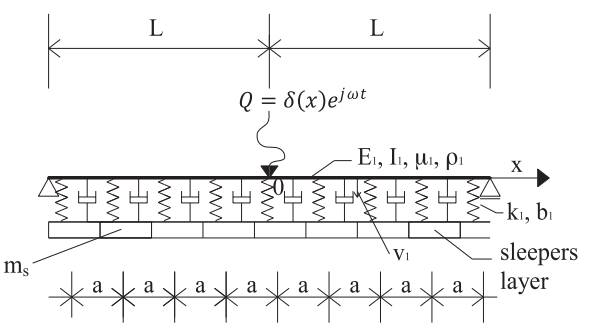

b)

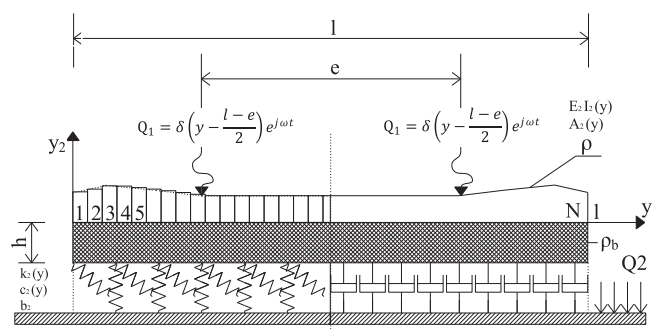

d)

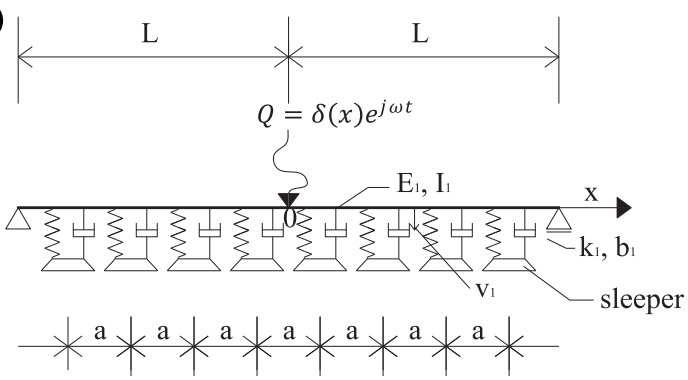

f)

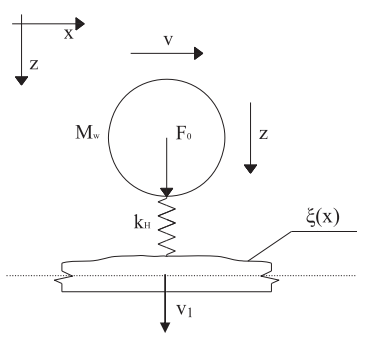

e)

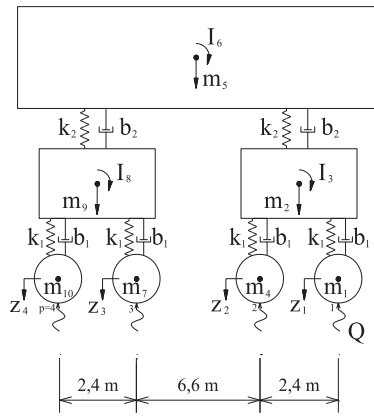

g)

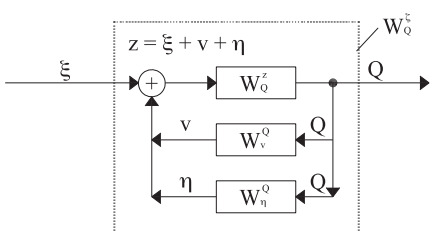

Fig. 2 The railway components model for numerical analysis

calculations results for vertical track receptance of rail deflection $v_{1}$ due to wheel contact forces $Q$.

Parameters used in calculation:

Sleeper SB8: $k_{2}=49.40 \mathrm{MPa} ; b_{2}=0.023 \mathrm{MPa} . \mathrm{s} ; c_{2}=0$; $h=0.45 \mathrm{~m} ; a=0.55 \mathrm{~m} ; \rho_{b}=0.0017 \mathrm{Mkg} . \mathrm{m}^{-3}$

Rail R65: $\quad L=12.12 \mathrm{~m} ; k_{1}=217000 \mathrm{MPa}$; $b_{1}=0.037 \mathrm{MPa} . \mathrm{s} ; E=210000 \mathrm{MPa}$; $I_{1}=3 \cdot 6 \cdot 10^{-5} \mathrm{~m}^{4} ; m_{r}=65 \mathrm{~kg} \cdot \mathrm{m}^{-1}$.

Vehicle: SKODA E 699; 10 degree of freedom; 4 axles; $k_{H}=$ $=1,5.10^{9} \mathrm{~N} \cdot \mathrm{m}^{-1}$; mass of axle: $1250 \mathrm{~kg}$; mass of bogie: $4750 \mathrm{~kg}$; mass of body casing: $23.500 \mathrm{~kg}$; bogie inertia moment: $5.5 .10^{3} \mathrm{~kg} \cdot \mathrm{m}^{2}$; bogie casing inertia moment: $5.10^{5} \mathrm{kgm}^{2} ; k_{1}=7.266 .10^{5} \mathrm{~N} \cdot \mathrm{m}^{-1}$ spring stiffness: $k_{2}=9.5 .10^{6} \mathrm{~N} . \mathrm{m}^{-1} ; k_{1}=7.266 .10^{5} \mathrm{~N} . \mathrm{m}^{-1}$; spring damping: $b_{1}=7.37 .10^{4} \mathrm{~N} \cdot \mathrm{m}^{-1} ; b_{2}=3.68 .10^{4} \mathrm{~N} \cdot \mathrm{m}^{-1}$; axle base: $2.8 \mathrm{~m}$; bogie base: $10.3 \mathrm{~m}$.

From proposed model it is possible to calculate also sleeper dynamic deflection and acceleration PSD as the input spectra
$S_{v_{2} v_{2}}(f)$ or PSD $S_{r_{i j}}(f)$ into the ballast and roadbed, see Fig. 4. To predict the level of ground vibration in the vicinity of railways it needs to calculate the response spectrum at distance point on the surface of a linear viscous - elastic half space $S_{w w}(f)$ via the frequency response function (FRF) - $H_{i k}(f)$ of the ground by a method involving integral transform [3].

\section{Prediction models for ground vibration propagation}

\subsection{The analytic-experimental approach}

The analytic-experimental approach proposes the test and the theory data combination to calculate the prediction level of ground vibration. In this process as an input signal can be used accelerations spectra (or spectral densities) derived from experimental data bank for authorized railway category with corresponding rail profile or accelerations spectrum $\bar{S}_{\text {wiw }}(f)$ measured at the nearest ground point to the track for individual case study. The frequency response function (transfer function) of the ground can be derived via experimental impulse seismic method (ISM) or cross-hole test 


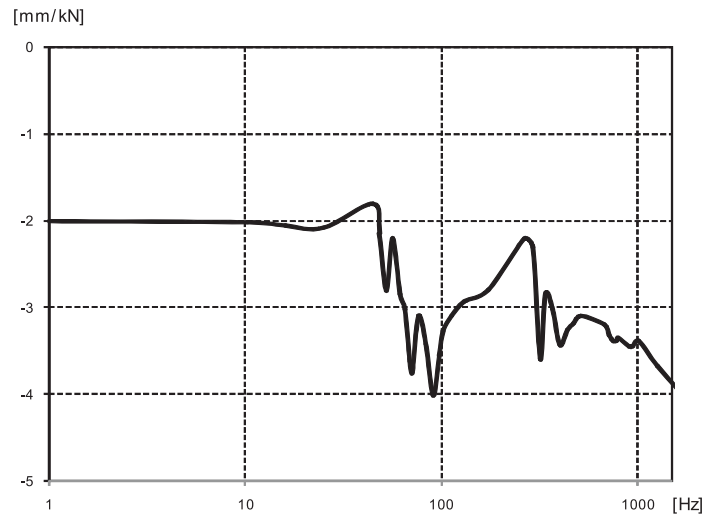

Fig. 3 The vertical track receptance of $v_{l}$

data [14], from which elastic and attenuation parameters of the ground can be obtained $[1,6]$, too. The measuring output response acceleration spectrum at the distance $S_{w w}(f)$ due to input accelerations spectrum $\bar{S}_{\text {ẅw }}(f)$ the FRF-H(f) can be derived, by equation [3], (see also Fig. 6)

$$
S_{\bar{w} \bar{w}}(f)=|H(f)|^{2} S_{\ddot{w} \ddot{w}}(f)
$$

\subsection{In - situ soil dynamic parameters tests}

Experimental tests at nearby building IBM region. To calculate prediction vibration level and dynamic response for projected new building in a new railway line area it was needed to know the building site soils dynamic parameters values and FRF. Therefore the in situ ISM tests in the IBM Data Centre building site were performed. The building site is situated in the same area in which the new Trans European Network (TEN-T) line is projected, too. After the both structures erection the distance between them will be approximately $20 \mathrm{~m}$. Hence the prediction of building vibration level and response spectra due to operating trains was required.

The building site is situated on level ground (sandy loam $3.5 \mathrm{~m}$ and gravel sand $-12.0 \mathrm{~m}$ ). This permits the ground to be modelled as a damped, viscoelastic half space. The viscoelastic model of soil simulation using the complex modulus conception $E^{*}=E\left(1+\delta_{E}\right)$ and $G^{*}=G\left(1+\delta_{G}\right)$ respectively, offers a very good approach $[2,11]$ to the actual soil behaviour $\left(E, G\right.$ and $\delta_{E} \approx$ $\approx \delta_{G}$ are real and imaginary components of complex modulus). The Raleigh's and shear waves propagation velocities $v_{R}$ in half space in this form are analysed in $[6,12]$. The experimental tests for the purpose of the evaluation of elastic and attenuation soil parameters are described in reference [6]. The $I B M$ building site layout and accelerometers and impact loading positions $\left(I_{i}\right)$ during the experimental tests are shown in Fig. 5.

The impulse test results are as follows:

- $v_{R}=145.10 \mathrm{~ms}^{-1} ; \delta_{G}=0.117 ; E_{0}=109.20 \mathrm{MPa} ; G_{0}=41.10$ $\mathrm{MPa}$,

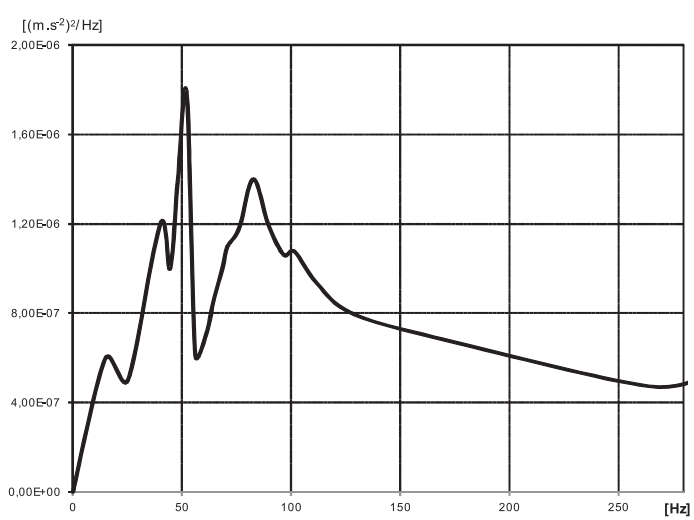

Fig. 4 The input PSD $G_{\vec{v}_{2} \vec{v}_{2}}(f)$

- the ISM test No. 5 spectral analysis results are plotted in Fig. 6

The calculation includes data: $\lambda_{R}=9.2 \mathrm{~m}$, (Raleigh's waves length); $\rho=1950 \mathrm{~kg} \cdot \mathrm{m}^{-3}$, (soil mass density); $\alpha=0.0398 \mathrm{~m}^{-1}$, (the attenuation coefficient obtained by standard deviations $\sigma(0), \sigma(y)$ of displacement amplitude vibration at the distance $l_{0}, l_{y}$ from source of excitation using the displacement power spectral densities $G_{i i}^{(0)}$ and $\left.G_{k k}^{(y)}\right)$.

\subsection{Vibration propagation process experimental spectral analysis}

An experimental study of ground vibration transmission from a railway was carried out in the same region as the impact tests, adjacent to the ZSR railway line Bratislava - Vienna, track No. 1 (No. 2) in the town district Bratislava Trnavka. The object of the experimental measurements was to find: spectral characteristics of the vibration components of the track near region soils by the accel-

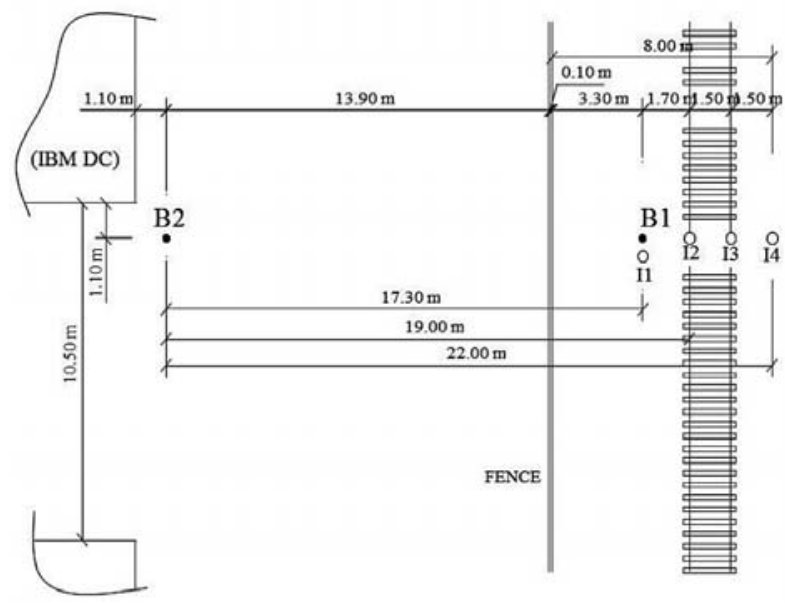

Fig. 5 Accelerometers and ISM (I1- I4) positions and projected building and railway site layout 

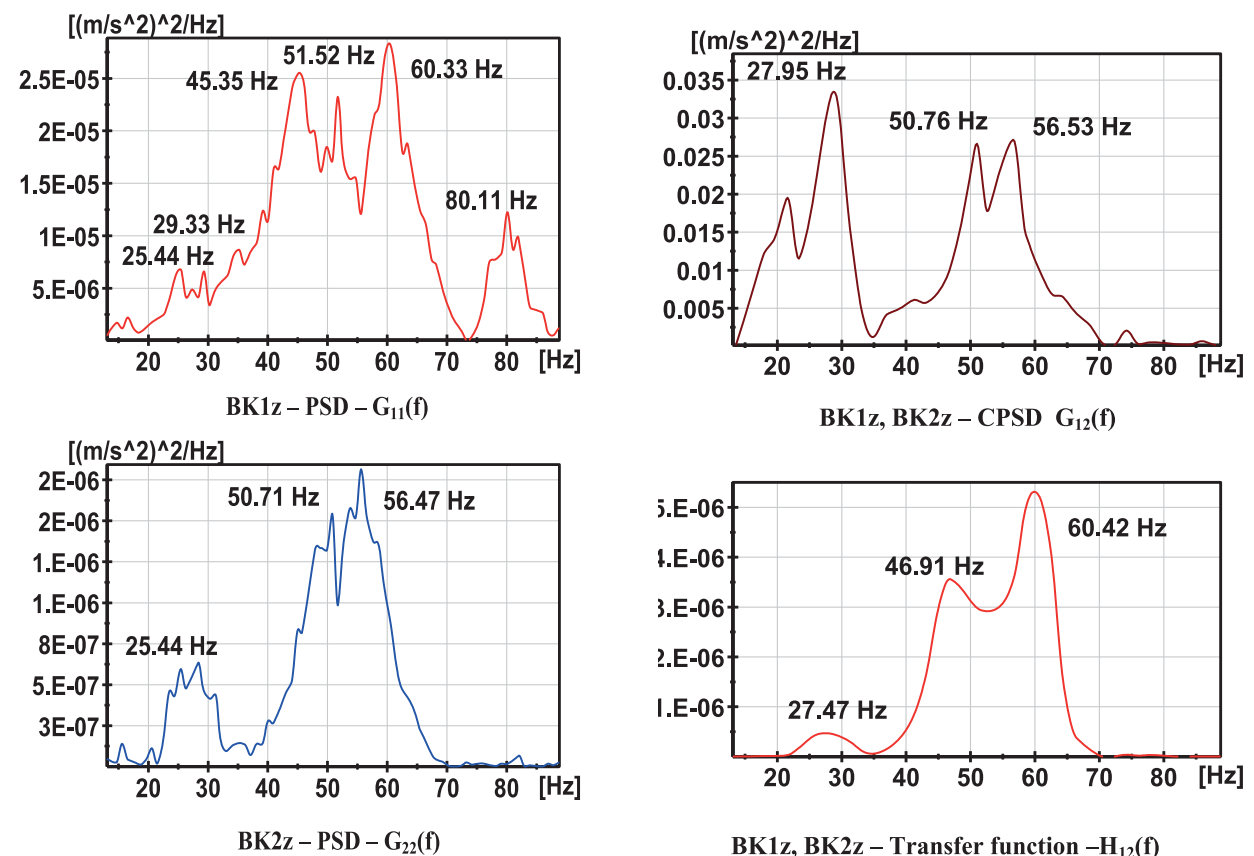

BK1z, BK2z - Transfer function $-\mathrm{H}_{12}(\mathrm{f})$

Fig. 6 The ISM5 test spectral analysis results at points B1 and B2

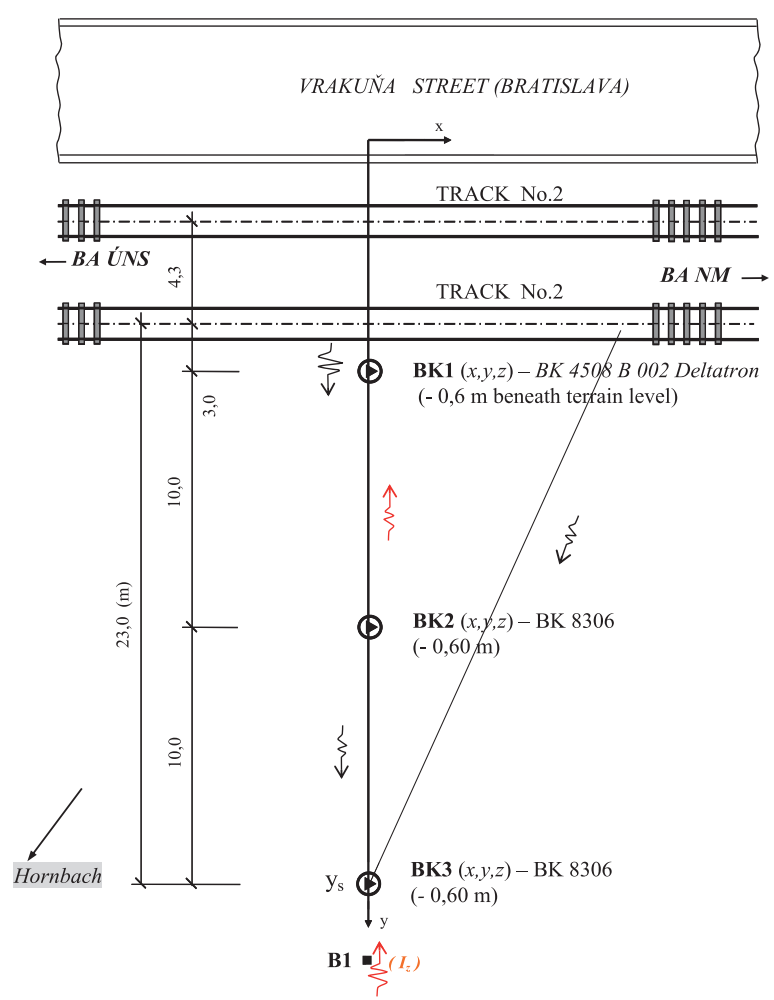

Fig. 7 The pickups and DLP positions in track region
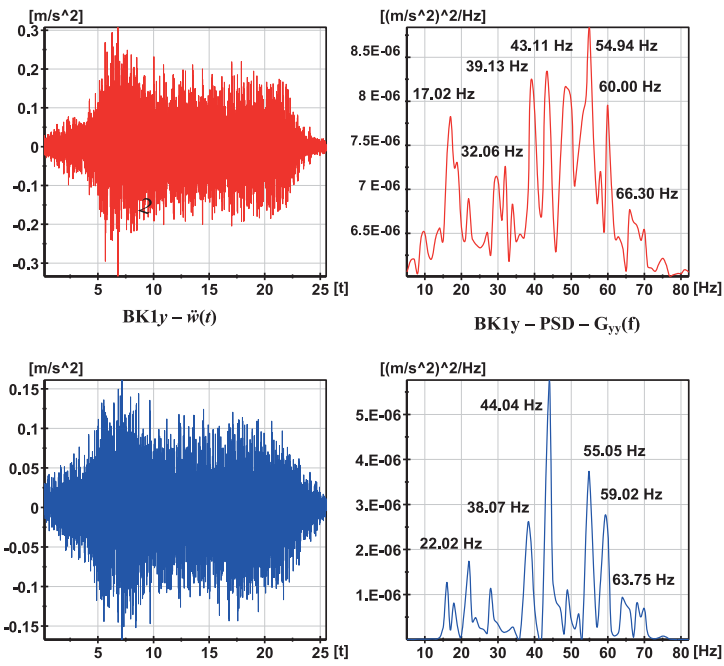

$\mathrm{BK} 1 \mathrm{x}-\ddot{w}(t)$

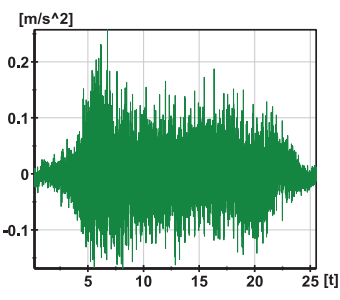

$\mathrm{BK} 1 \mathrm{z}-\ddot{w}(t)$

BK1x - PSD $-\mathbf{G}_{\mathbf{x x}}(\mathbf{f})$

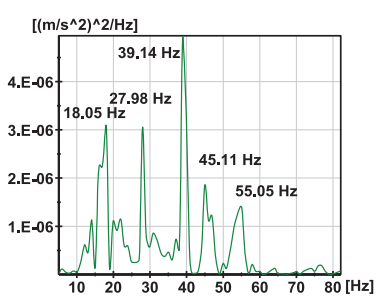

BK1z - PSD - $\mathbf{G}_{z}(\mathrm{f})$

Fig. 8 The accelerations time histories and PSD at point BK1 
eration power spectral densities $G_{i i}(f), G_{k k}(f), G_{i k}(f)$. The pickups positions are shown in Fig. 7. The roadbed and ground accelerations of the vibrations were recorded using a portable notebook computer with $N I$ software and hardware facilities. The test experimental procedure in details is described in [6]. As an example of the train induced vibrations accelerations spectral analysis results (PSD) in the ground at measured point BK1 are plotted in Fig. 8.

\section{Dynamic response of the building structure prediction}

To calculate the prediction vibration level and structure dynamic response of the projected new building structure situated at the nearest area of the projected new railway line it was needed to know the building site soils dynamic parameters, site geological medium FRF and also the representative input accelerations spectra similar to expected real train spectra in future traffic [6]. For calculation of expected structure dynamic response it was used: (i) PSD $G_{i i}(f)$ of ground acceleration at the track nearest region (Fig. 7) with similar geological medium data as site medium (ZSR - railway line Bratislava - Vienna, track No. 1 (No. 2) in the town district Bratislava Trnavka) as input spectra (see Fig. 8), (ii) The halfspace transfer function $H_{i k}(f)$ of the building site geological medium obtained by ISM tests (Fig. 6) and (iii) project of building structure. The response PSD - $G_{k k}(f)$ of the halfspace point at the projected structure foundations location were calculated and plotted, (Fig. 11).

In the next step these spectra were used as the input spectra for the expected building structure dynamic response calculation due to train. The dynamic response of the building structure numerical calculation was carried out by the Visual Fea program package.

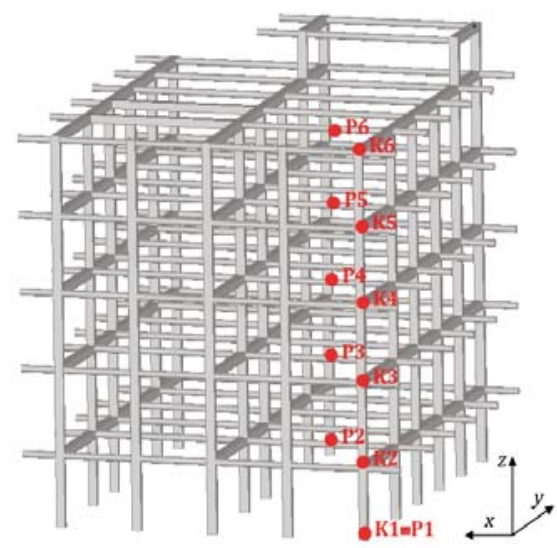

Fig. 9 FEM model render
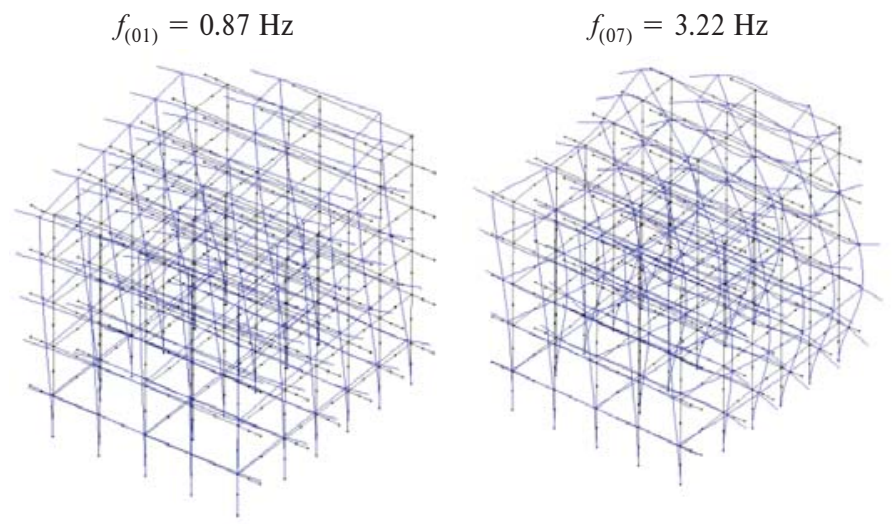

Fig. 10 Structure natural modes
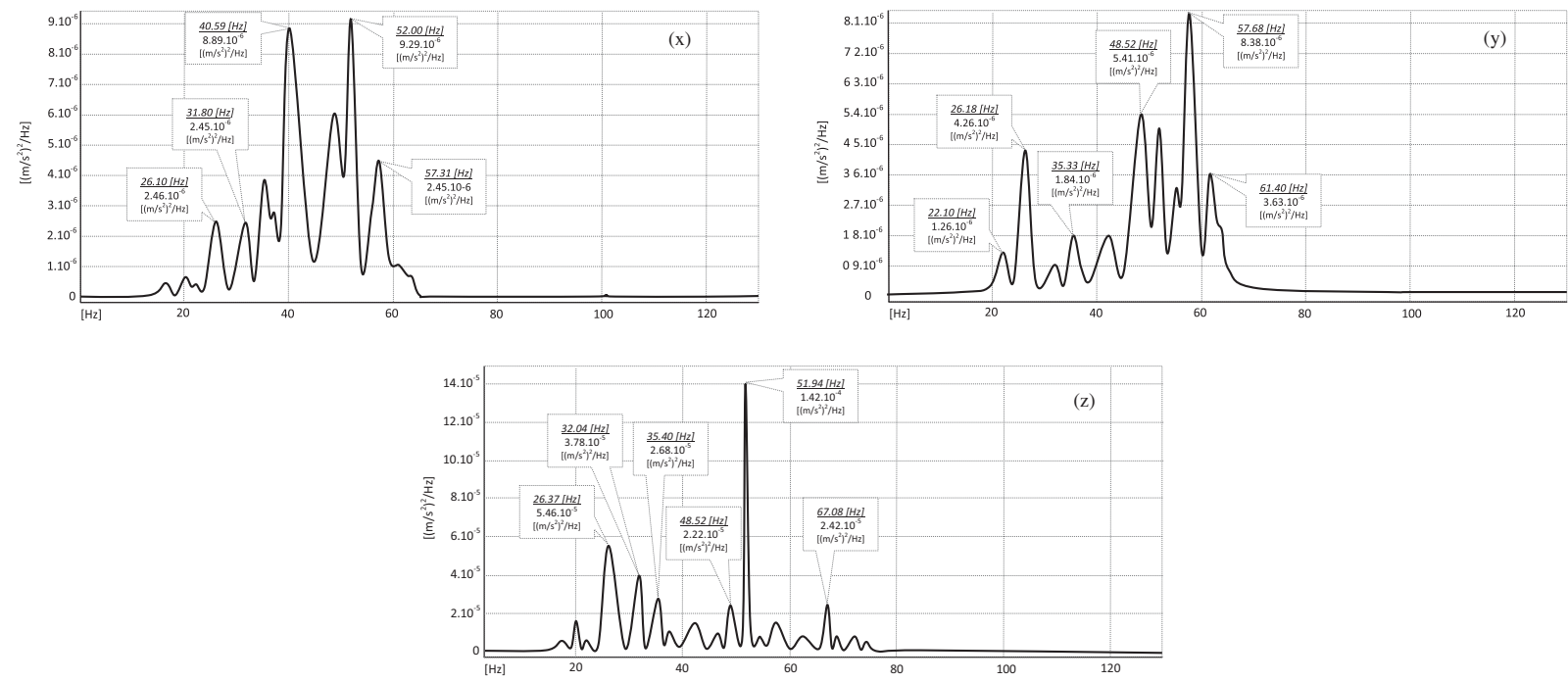

Fig. 11 The input acceleration PSD $-G_{11}(f)$ to foundation at point $\mathrm{K1} / 10-130 \mathrm{~Hz}$ 

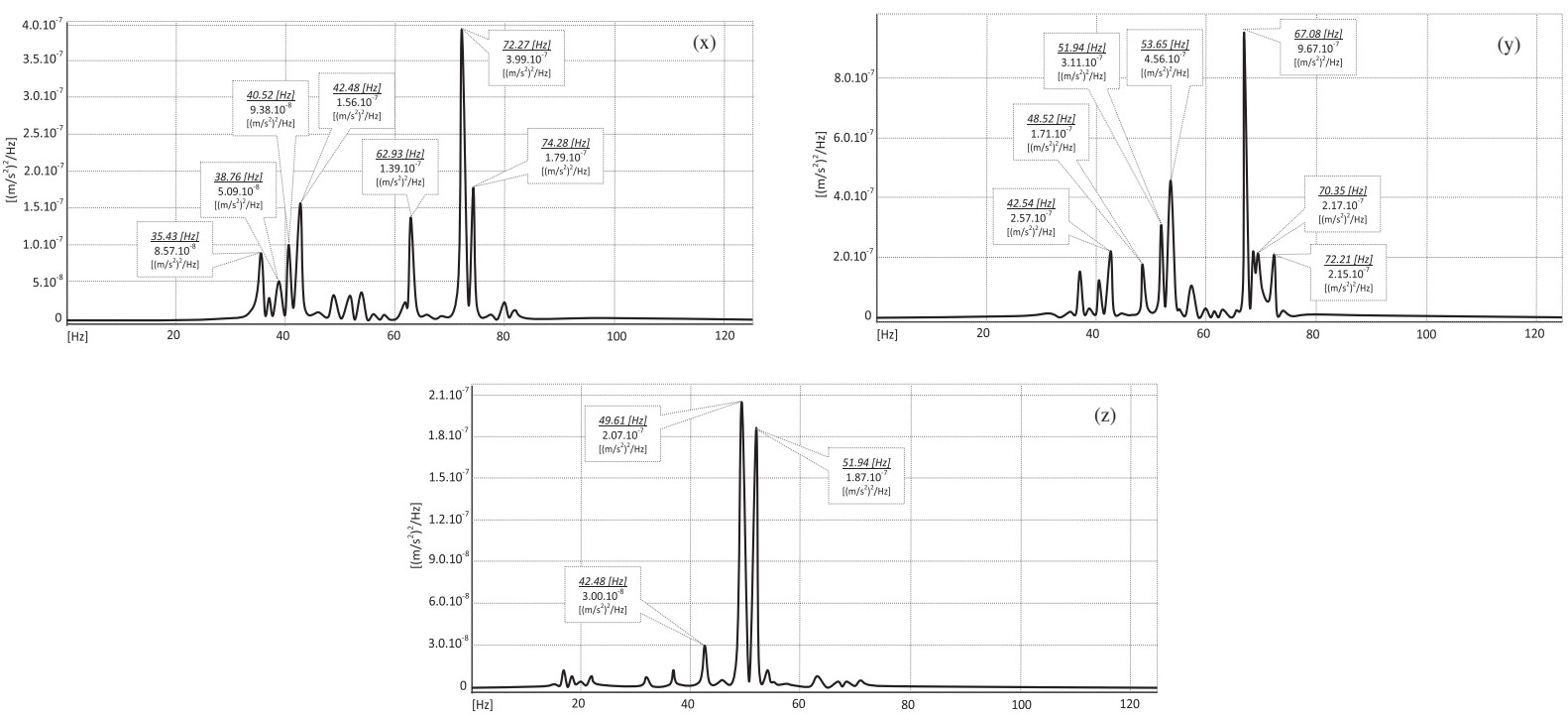

Fig. 12 The response acceleration $P S D-G_{66}(f)$ of roof at point $\mathrm{K} 6 / 10-130 \mathrm{~Hz}$

The calculated values of natural frequencies and natural modes were applied for the building structure dynamic response calculations. The structure FEM model render and natural modes examples are shown in Figs. 9 and 10. Also the building structure dynamic response calculations were carried out in frequency domain. The spectral analysis was divided into two parts: low frequency band $(0-10 \mathrm{~Hz})$ and higher frequency band $(10-130 \mathrm{~Hz})$.

The low frequency band spectral analysis gives the basic natural frequencies vibration rangewhich enables to predict possible resonance effects of structure vibration due to traffic. Because of the vibration sensitive technologies installing in the IBM $D C$ building the power spectral densities are determined in the structure relevant points (over columns and in the middle of beam spans) for the structure dynamic response considering in the range of frequency band $10-130 \mathrm{~Hz}$. The higher frequency spectral analysis band is mainly required for vibration level assessment on monitored frequency according to the IBM Corporate Standard C-S1-9711002, 1990-03 requirements. The accelerations PSD and displacements extreme and RMS values were numerically calculated at selected render points (K1...K6, P1...P6) in the three vibration directions $x, y, z$. The results of the IBM DC building structure dynamic response calculations are presented in detail by Bencat [6]. The adequate input load acceleration PSD - $G_{11}(f)$ into the foundation structure (render point $\mathrm{K} 1$ ) for $x, y$ and $z$ vibration direction are plotted in Fig. 11 and the response acceleration PSD - $G_{66}(f)$ of the building roof structure (render point K6) is plotted in Fig. 12.

\section{Conclusions}

Based on the results presented in this paper the following conclusions can be drawn:
- The numerical prediction model can account for many parameters of the train-track-soil interaction problem. Final products of the numerical calculations are vehicle, rail, sleeper, railpads and ballast frequency response functions using spectral density functions of the rail roughness. To predict the level of ground vibration in the vicinity of railways it needs to calculate the response spectrum at distance point on the ground surface $S_{w w}(\omega)$ via the FRF - $H_{i k}(f)$ of the ground by a method involving integral transform.

- The numeric - experimental prediction model. The numeric-experimental approach process proposes the test and the theory data combination to calculate the prediction level of ground vibration.

- The frequency response function - $H(f)$ of the ground for the case study was derived via experimental impulse seismic method ISM test data, from which elastic andattenuation parameters of the ground were obtained, too.

The calculation results of the predicted IBM Data Centre building dynamic response using relevant input experimental data as the case study example are introduced, too. The relevant calculated data values following from spectral and amplitude analysis of the predicted building dynamic response (spectral picks limit, vibration levels, etc.) were compared with relevant standards prescription values and criteria (IBM Corporate Standard C-S1, Slovak Standard STN 73 0036, STN EN 1998 - 1/NA/Z1 (EC8), etc.). From these comparisons it follows that all standards prescription values and criteria in future will be fulfilled.

\section{Acknowledgement}

We kindly acknowledge the research project VEGA, Nr.GI/ 0169/12 granted by Scientific Grant Agency of the Slovak Republic Ministry of Education. We should also like to thank the Civil Engineering Faculty - University of Zilina for additional feed in this field research activity. 


\section{References}

[1] BENCAT, J.: Microtremor from Railway Traffic, Proc. of the $8^{\text {th }}$ Intern. Conf. on Computational Structures Technology, Gran Canaria, Sept.2006. Civil - Comp Press, Stirlingshire, Scotland, 2006.

[2] BENCAT, J.: Microtremor Due to Traffic, Research report A - 4 - 92/b, UTC Zilina, 1992 (in Slovak).

[3] BENDAT, J. S., PIERSOL, A. G.: Engineering Applications of Correlation and Spectral Analysis, Wiley \& Sons, New York, 1993.

[4] FUJIKAKE, T.: A Prediction Method for the Propagation of Ground Vibration from Railway Trains, J. of Sound and Vibration, 111(2), pp. 357-360, 1986.

[5] FORD, R.: The Production of Ground Vibrations by Railway Trains, J. of Sound and Vibration 116(3), 585-589, 1987.

[6] BENCAT, J., et al.: Studies on the TEN-T Railway Traffic Effects on IBM Data Centrum - ST Building in Bratislava, Report PC 16/SvF/2009, UTC SvF Zilina, 2009.

[7] GRASSIE, S., COX, S.: The Dynamic Response of Railway Track with Flexible Sleepers to High Frequency Vertical Excitation, Proc. of the Institution of Mechanical Engineers 198D(7), 117-124, 1984.

[8] GRUNDMANN, H., LIEB, M., TROMMER, E.: The Response of a Layered Half-space to Traffic Loads Moving Along its Surface, Archive of Applied Mechanics 69,1999.

[9] KNOTHE, K., WU, Y.: Receptance Behaviour of Railway Track and Subgrade, Archive of Applied Mechanics 68, 457-470, 1998.

[10] KAWECKY. J., KOZIOL, K., STYPULA, K.: Design of Rail Track Including the Influence of Vibration on People in Buildings, Proc. of the $11^{\text {th }}$ Conf. on Computational Structures Technology, Dubrovnik, September, 2012, Civil-Comp Press, Edinburgh, UK, 2012, Paper 176, (doi:10.4203/ccp.99.176).

[11] BENCAT, J., CIBULKA M., HRVOL, M.: Evaluation of the Soil Elastic Modules by Means of Box Tests, Communications - Scientific Letters of the University of Zilina, No. 2, 2008,

[12] BENCAT, J., PAPAN, D.: Buildings Structure Response Due to Railway Traffic Prediction Model, Proc. of $18^{\text {th }}$ Intern. Congress on Sound \& Vibration, ICSV18, Rio de Janeiro, July, 2011.

[13] TUREK, J.: The Interaction Model of the Vehicle-Track System, Research report 245/92 VUZ Prague, 1992.

[14] IZVOLT, L., KARDOS, J.: Influence of Parameters of Railwway Track Construction on Vertical Dynamic Interaction Vehicle/Track, Communications - Scientific Letters of the University of Zilina, No. 3, 2011. 